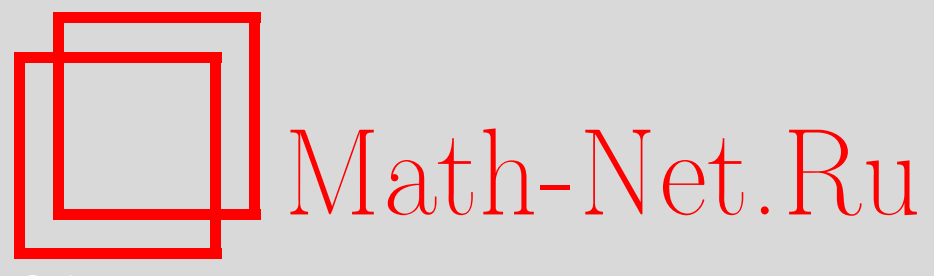

В. П. Маслов, Плотности решеток, соответствующих пространствам положительной, отрицательной и вариационной размерностей, и их применение к временным рядам, Матем. заметки, 2007, том 81, выпуск 2, 251264

DOI: https://doi.org/10.4213/mzm3552

Использование Общероссийского математического портала Math-Net.Ru подразумевает, что вы прочитали и согласны с пользовательским соглашением http://www . mathnet.ru/rus/agreement

Параметры загрузки:

IP : 52.205 .19 .152

26 апреля 2023 г., $14: 25: 51$

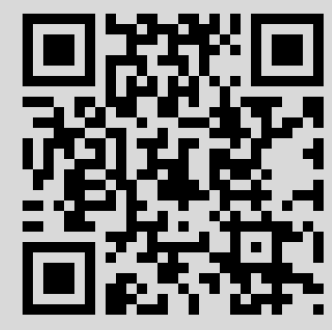


Том 81 выпуск 2 февраль 2007

УДК 519

\section{Плотности решеток, соответствующих пространствам положительной, отрицательной и вариационной размерностей, и их применение к временным рядам}

\section{В. П. Маслов}

В работе доказывается общая теорема о распределении типа Бозе-Эйнштейна. Понятие размерности и плотности решеток применяется с помощью этой теоремы к колеблющимся временным рядам.

Библиография: 15 названий.

В настоящей работе мы ставим три принципиальных вопроса. Вначале приведем эвристические соображения.

1. Какова связь между непрерывным и дискретным: между непрерывным пространством и отвечающей ему решеткой - скелетом этого пространства, его рамкой. Отрезки трех мерной решетки можно выпрямить в прямую, решетка по обычным понятиям одномерна.

Как отобразить на решетку это пространство? Если ставить вопрос в более общей математической форме: как отобразить на натуральный ряд (точек) компакт хаусдорфовой размерности $D$ ?

На отрезке $0 \leqslant x \leqslant 1$ существует шкала $0 \leqslant D \leqslant 1$ канторовой пыли с мерой Хаара, равной $x^{D}$ отрезка $(0, x)$, подобного всему данному множеству канторовой пыли. Прямое произведение этой шкалы на евклидов куб размерности $k-1$ даст всю шкалу $k+\sigma$, где $k \in Z, \sigma \in(0,1)$.

Пусть задан натуральный ряд $i=1,2, \ldots$. Сопоставим точке $i$ вес, отвечающий положительной размерности $D$ :

$$
\rho_{i}(D)=\frac{\Gamma(D+i-1)}{\Gamma(D) \Gamma(i)} .
$$

В силу формулы Эйлера [1]

$$
\Gamma(D)=\lim _{i \rightarrow \infty} \frac{(i-1) !}{D(D+1) \cdots(D+i-1)} i^{D},
$$

вес $\rho_{i}(D)$ при $i \rightarrow \infty$ стремится к $i^{D-1}$, т.е. к весу, отвечающему [2] в непрерывном случае размерности $D$. Отсюда

$$
\sum_{i=k}^{k+s} \rho_{i}(D)
$$

(C) В. П. МАслов, 2007 
при $D<1$ и $k \rightarrow \infty$ отвечает мере Хаара для канторовой пыли хаусдорфовой размерности $D$ "отрезка" длины $s$.

Можно сказать, что $\rho_{i}(D)$ - "квантованная" плотность пространства хаусдорфовой размерности $D$.

2. Второй вопрос - вопрос о пространствах отрицательной размерности. Дирак следующим образом объяснял парадокс, заключающийся в наличие отрицательных энергий в уравнении, которое он открыл, конструируя релятивистское уравнение электрона для объяснения его спина. Он предположил, что все отрицательные уровни энергии заполнены электронами. Когда электрон покидает отрицательный уровень, образуется дырка - позитрон, которая ведет себя как электрон с положительным зарядом. Этим удивительным рассуждением он предсказал существование позитрона.

В наших работах [3]-[6] дано следующее определение отрицательной размерности пространства.

ОПРЕДЕЛЕНИЕ (Общее определение пространств отрицательной размерности). Пусть имеется компакт $M_{t_{0}}$ хаусдорфовой размерности $t_{0}$, являющийся элементом $t$-параметрической шкалы вложенных друг в друга компактов $0<t<\infty$. Две шкалы будем называть эквивалентными относительно $M_{t_{0}}$, если у них совпадают компакты при $t \geqslant t_{0}$. Будем говорить, что компакт $M_{t_{0}}$ является дыркой в этом эквивалентом множестве шкал, а $-t_{0}$ есть отрицательная размерность этого класса эквивалентности.

"Квантование" пространств с отрицательной размерностью проводится точно так же [4], как и с положительной, по той же формуле (1), поскольку Г-функция аналитически продолжается для отрицательных $D$. Если $D$ - целое отрицательное, то при $i=1, \ldots,|D+1|$ имеют место простые полюса, которые являются дискретным аналогом инфракрасной катастрофы. Можно также считать эти точки конденсатом. Поэтому имеют смысл только значения плотности при $i>|D|$, и они равны

$$
\rho_{i}=\frac{1}{i(i-1)(i-2) \cdots(i-D-1)} .
$$

Если $D$ - отрицательное нецелое, то при $i>|D|$

$$
\rho_{i}=\frac{\Gamma(i+D-1)}{(i-1) !},
$$

а при $i<D \rho_{i}$ меняют знаки.

Однако пространства с отрицательной размерностью, если нет "непрерывного" аналога, не так просто распознать. Приведем важный пример.

В наших предыдущих работах мы применили метод квантовой статистики к лингвостатистике [7].

Каждому слову в частотном словаре соответствует число его встречаемости в исходном корпусе текстов. Некоторым словам может соответствовать одно и то же число встречаемости в тексте.

Ранг слова (т.е. его порядковый номер в частотном словаре) отсчитывается от слова $A_{1}$ с самым большим числом встречаемости в заданном корпусе текстов. Обозначим число слов с одинаковым числом встречаемости $i$ через $N_{i}$. Тогда, если 
$A_{m}$ - последнее слово в списке слов одинаковой встречаемости $m$ (слова с одинаковым числом встречаемости могут быть упорядочены произвольным образом), то ранг $r_{m}$ слова $A_{m}$ равен

$$
r_{m}=\sum_{i=m}^{s} N_{i}
$$

Аналогия между бозе-частицами на уровне энергии осциллятора $\lambda_{i}=i$ и словами с частотой (числом) встречаемости $i$ заключается в том, что слова с одной и той же частотой встречаемости мы можем упорядочивать как угодно: в алфавитном, обратном алфавитном или в любом другом порядке. Нумерация рангов (номеров) слов внутри одной частоты (числа) встречаемости произвольна. В этом смысле слова неразличимы и подчиняются бозе-статистике.

Однако в рассматриваемых подходах есть различие. В частотном словаре считают число встречаемости каждого слова, а затем упорядочивают слова, начиная от наиболее часто встречающихся.

Когда не было компьютеров, человеку было сложно вычислять число слов с одинаковой частотой встречаемости. Человек способен, посмотрев на страницу как на картинку, определить на ней искомое слово по его графическому образу в стольких местах, во скольких оно встречается. Человек смотрит на страницу текста в этом случае как на фотографию, не вникая в смысл. Аналогично, если человек ищет определенную фамилию в длинном списке фамилий людей, принятых в институт, он не прочитывает все фамилии подряд, а глазами находит (или не находит) нужное имя.

Глаз привыкает распознавать нужное изображение, и эта способность обостряется с увеличением просматриваемого материала: чем больше страниц он просматривает, тем легче находит нужный графический образ. Поэтому при ручном подсчете проще было, не читая текст, распознавать на странице искомое слово и вычеркивать его карандашом, считая при этом, сколько раз оно встретилось. Такая процедура повторяется для каждого следующего слова уже по тексту с вычеркнутыми словами ("дырками"), что облегчает поиск. Иначе говоря, эта процедура заключается в распознавании образа данного слова, как мы распознаем, например, в лесу нужный нам гриб, не перебирая все подряд, что растет на земле. Обычный компьютер это делает перебором, а квантовый (см. [8]), распознавая образ.

Но и для обычного компьютера число операций для нахождения частоты встречаемости слова меньше, чем для нахождения числа слов в тексте с данной частотой встречаемости.

Можно сказать, что число грибов, которые мы собрали (отобрали у леса), это число дырок, которые мы оставили в лесу. Точно так же слова, которые мы "выловили" из текста описанным выше образом, - это аналог не частиц, а дырок. Поэтому и ранг слов лингвисты считают с другого конца, по сравнению с тем, как это делали бы физики. Физики считали бы частицы, начиная с нижнего уровня, а дырки отсутствующие электроны, начиная с верхнего.

Поэтому слова в частотном словаре ассоциируются не с частицами, а с дырками. Соответственно, размерность в распределении частотного словаря нужно выбирать “дырочную”, отрицательную или нулевую. 
И это, возможно, ответит на вопрос о том, как правильно определять размерность в дискретном варианте, - ответ в духе идей Колмогорова. Если длина алгоритма определения дырочной размерности много меньше, чем длина алгоритма, определяющего положительную размерность, то это - дырочная размерность.

Пусть $E$ - число словоупотреблений в исследуемом массиве текстов (длина массива); $N$ - число слов в отвечающем этому массиву словаре. Тогда $\omega^{0}=E / N-$ средняя частота встречаемости слова в тексте.

Если $\omega_{i}$ - число встречаемости $i$-го слова в тексте, то будем называть относительной частотой $\bar{\omega}_{i}=\omega_{i}-\omega^{0}$, т.е. для всех частот встречаемости больших средней частоты $\omega^{0}=E / N \bar{\omega}_{i}$ положительно, а для меньших - отрицательно.

Последовательности слов в тексте сопоставим последовательность их относительных частот $\bar{\omega}_{i}$ и рассмотрим кумулятивную сумму $f_{k}=\sum_{i=1}^{k} \bar{\omega}_{i}$.

Полагаю, что показатель Гёльдера для $f_{k}$ равен $1 / 2$. В этом случае размерность текста, как это следует из приводимой ниже формулы (12), будет нулевой и полюс (конденсат) будет при $i=1$, что отвечает всем известным словарям.

3. Третий вопрос связан с показателем Гёльдера, который можно выразить через вариационную размерность. Понятие вариационной размерности идейно близко к хаусдорфовой размерности. При этом в последнее время в связи с экономическими задачами оно применяется к временному ряду, т.е. в основном к дискретному случаю.

Мы приведем определение показателя Гёльдера, данное Шафером и Вовком [9], в терминах нестандартного анализа.

Рассмотрим произвольный интервал $[0, T]$ (стандартных) действительных чисел, $T>0$, и пусть $N$ - бесконечно большое нестандартное натуральное число. Эти числа далее фиксированы. Обозначим $d t=T / N$. Основное множество

$$
\mathscr{T}=\{n d t \mid 0 \leqslant n \leqslant N\}
$$

содержит все стандартные числа из интервала $[0, T]$, а также те нестандартные действительные числа, которые отличаются от них на бесконечно малые величины.

Пусть $f$ - произвольная функция на $\mathscr{T}$ и $t \in \mathscr{T}-\{T\}$. Положим $d f(t)=f(t+d t)-$ $f(t)$.

ЛЕмма 1. Пусть $f$ - непрерывная (возможно принимающая нестандартные значения) функция на $\mathscr{T}, p \in[1, \infty)$ - действительное число и пусть

$$
\operatorname{var}_{f}(p)=\sum_{t \in \mathscr{T}-\{T\}}|d f(t)|^{p} .
$$

Тогда существует единственное действительное число $1 / H(f) \in[0,1]$ такое, что

1) $\operatorname{var}_{f}(p)$ - бесконечно большое при $1 \leqslant p<1 / H(f)$;

2) $\operatorname{var}_{f}(p)$ - бесконечно малое при $p>1 / H(f)$.

Число $H(f)$ будем называть показателем Гёльдера, а $1 / H(f)$ - вариационной размерностью.

Если $H(f)=1 / 2$, то функция $f$ является винеровской.

Мы воспользуемся соотношением между гладкостью функций и поведением на бесконечности ее преобразования Фурье, о котором мы говорили в [4]. 
Известна связь между гёльдоровским показателем $H$ стационарного случайного процесса и показателем $\alpha$ степенного убывания его спектра.

Пусть сигнал $f(t)$ стационарен, так что $\langle f(t)\rangle \equiv\langle f\rangle=$ const, и имеет гёльдеровский показатель $H$, так что

$$
|f(t+s)-f(t)|^{2} \sim s^{2 H}, \quad 0<H<1 .
$$

Тогда для автокорреляционной функции $F(s)$

$$
F(s)=\langle f(t+s) f(t)\rangle-\left\langle f^{2}\right\rangle
$$

получаем

$$
F(0)-F(s) \sim s^{2 H}
$$

По теореме Винера-Хинчина спектральная плотность $J(\omega)$ является преобразованием Фурье автокорреляционной функции:

$$
J(\omega)=\int F(s) \exp (i \omega s) d s=\int F(s) \cos \omega s d s
$$

и, соответственно,

$$
F(s)=\frac{1}{2 \pi} \int J(\omega) \exp (-i \omega s) d \omega=\frac{1}{2 \pi} \int J(\omega) \cos \omega s d \omega .
$$

Поскольку функция $F(s)$ четна, имеем

$$
F(0)-F(s)=\frac{1}{2 \pi} \int J(\omega)(1-\cos \omega s) d \omega=\frac{1}{\pi} \int J(\omega) \sin ^{2} \frac{\omega s}{2} d \omega .
$$

Пусть $J(\omega)$ убывает на бесконечности как $|\omega|^{-\alpha}$. Тогда

$$
F(0)-F(s) \sim \int|\omega|^{-\alpha} \sin ^{2} \frac{\omega s}{2} d \omega=s^{\alpha-1} \int|\omega s|^{-\alpha} \sin ^{2} \frac{\omega s}{2} d(\omega s) .
$$

Заменяя в последнем интеграле переменную на $u=\omega s$, видим, что зависимость от $s$ остается только в множителе перед интегралом. Таким образом, $s^{2 H} \sim F(0)-F(s) \sim$ $s^{\alpha-1}$ или

$$
H=\frac{1}{2}(\alpha-1), \quad \alpha=2 H+1 .
$$

Для колмогоровской турбулентности $H=1 / 3, D=-5 / 3$.

Поскольку мы оговорили, что переменная $t N$ принимает дискретные значения $t=1,2, \ldots, N$, то в силу принципа двойственности Понтрягина, или, как это принято в физической литературе, по теореме Котельникова "минимальный" ряд Фурье функции, совпадающий в точках $t=1,2, \ldots, N$, состоит из $N$ членов.

Из (12) следует, что если $H$ полуцелое, то $\alpha$ целое. Но как говорилось, $H=1 / 2$ отвечает винеровскому процессу (броуновскому движению), а это означает в силу (1), что у функции $\rho_{i}(-2)$ в точках $i=1$ и $i=2$ простые полосы. Таким образом, для дискретного броуновского движения первые два члена $a_{1}$ и $a_{2}$ преобразования Фурье обращаются в бесконечность. Значит, имеет место инфракрасная катастрофа. 
Если же $H \neq 1 / 2$, то удобнее находить $H$ из отношения $a_{2} / a_{1}$ первых двух членов ряда Фурье или просто использовать это отношение вместо вычисления $H$ как показатель отклонения от винеровского процесса.

Как рассматривалось в [3], относительно значений случайной величины $\lambda_{1}, \ldots, \lambda_{s}$ устанавливается упорядоченность по их величине. Среди чисел $\lambda_{1}, \ldots, \lambda_{s}$ могут быть и равные. В последнем случае их объединяют, складывая при этом отвечающие им "вероятности", т.е. отношение числа "попаданий" на $\lambda_{i} \mathrm{~K}$ общему числу испытаний. Число равных $\lambda_{i}: \lambda_{i}=\lambda_{i+1}=\lambda_{i+k}$ будем называть кратностью $q_{i}$ значения $\lambda_{i}$. Если размерность равна $D$, то

$$
q_{i}=\frac{\rho_{i}(D)}{\min _{1 \leqslant i \leqslant s} \rho_{i}(D)} .
$$

В нашем рассмотрении к бесконечности стремится как число испытаний $N$, так и $s$.

Пусть $N_{i}$ - число "выпадений” значения $\lambda_{i}: \lambda_{i}<\lambda_{i+1}$, тогда

$$
\sum_{i=1}^{s} \frac{N_{i}}{N} \lambda_{i}=M
$$

где $M$ - математическое ожидание.

Кумулятивная вероятность $\mathscr{P}_{k}$ есть сумма первых $k$ вероятностей в последовательности $\lambda_{i}$ : $\mathscr{P}_{k}=(1 / N) \sum_{i=1}^{k} N_{i}$, где $k<s$. Обозначим $N P_{k}=B_{k}, \bar{\lambda}=$ $\sum_{i=1}^{s} q_{i} \lambda_{i} / Q, Q=\sum_{i=1}^{s} q_{i}$.

Задача распадается на два случая:

Случай 1)

$$
E<\bar{\lambda} N
$$

Случай 2)

$$
\bar{\lambda} N \leqslant E \leqslant \lambda_{\max } N .
$$

Для первого случая имеет место нижеследующая теорема.

Если все варианты, для которых

$$
\sum_{i=1}^{s} N_{i}=N
$$

и

$$
\sum_{i=1}^{s} N_{i} \lambda_{i} \leqslant E
$$

равноценны (равновероятны), то [10]-[12] большинство вариантов будет скапливаться около следующей зависимости “кумулятивной вероятности" $B_{l}\left\{N_{i}\right\}_{i=1}^{l}=\sum_{i=1}^{l} N_{i}$,

$$
\sum_{i=1}^{l} N_{i}=\sum_{i=1}^{l} \frac{q_{i}}{e^{\beta^{\prime} \lambda_{i}-\nu^{\prime}}-1},
$$

где $\beta^{\prime}$ и $\nu^{\prime}$ определяются из условий

$$
B_{s}=N, \quad \sum_{i=1}^{s} \frac{q_{i} \lambda_{i}}{e^{\beta^{\prime} \lambda_{i}-\nu^{\prime}}-1}=E,
$$


если $N \rightarrow \infty$ и $s \rightarrow \infty$.

Дополнительно к [3] мы предположим, что $a_{1} N \leqslant Q \leqslant a_{2} N, a_{1}, a_{2}=$ const, $Q=$ $\sum_{i=1}^{s} q_{i}$.

Введем обозначения: $\mathscr{M}$ - множество всех наборов $\left\{N_{i}\right\}$, удовлетворяющих условиям (14) и (15); $\mathscr{N}\{\mathscr{M}\}$ - число элементов множества $\mathscr{M}$.

Теорема 1. Пусть все варианты наборов $\left\{N_{i}\right\}$ таких, что выполняются условия (14) и (15), равновероятны. Тогда число вариантов $\mathscr{N}$ наборов $\left\{N_{i}\right\}$, удовлетворяющих (14) и (15), для которых выполняется дополнительное соотношение

$$
\left|\sum_{i=1}^{l} N_{i}-\sum_{1}^{l} \frac{q_{i}}{e^{\beta^{\prime} \lambda_{i}-\nu^{\prime}}-1}\right| \geqslant N^{(3 / 4+\varepsilon)},
$$

менъше чем $c_{1} \mathscr{N}\{\mathscr{M}\} / N^{m}$, где $c_{1}$ и $m$-любие числа, $\sum_{i=1}^{l} q_{i} \geqslant \varepsilon Q, \varepsilon-$ сколь угодно малое.

ДоКАЗАТЕЛЬСТво теоремЫ 1 . Пусть $\mathscr{A}$ - подмножество $\mathscr{M}$, удовлетворяющее условию

$$
\begin{gathered}
\left|\sum_{i=l+1}^{s} N_{i}-\sum_{i=l+1}^{s} \frac{q_{i}}{e^{\beta \lambda_{i}-\nu}-1}\right| \leqslant \Delta, \\
\left|\sum_{i=1}^{l} N_{i}-\sum_{i=1}^{l} \frac{q_{i}}{e^{\beta^{\prime} \lambda_{i}-\nu^{\prime}}-1}\right| \leqslant \Delta,
\end{gathered}
$$

где $\Delta, \beta, \nu$ - некоторые действительные числа, не зависящие от $l$.

Обозначим

$$
\begin{aligned}
\left|\sum_{i=l+1}^{s} N_{i}-\sum_{i=l+1}^{s} \frac{q_{i}}{e^{\beta \lambda_{i}-\nu}-1}\right| & =S_{s-l}, \\
\left|\sum_{i=1}^{l} N_{i}-\sum_{i=1}^{l} \frac{q_{i}}{e^{\beta^{\prime} \lambda_{i}-\nu^{\prime}}-1}\right| & =S_{l} .
\end{aligned}
$$

Очевидно, что если $\left\{N_{i}\right\}$ множество всех наборов $\left\{N_{i}\right\}$, то

$$
\mathscr{N}\{\mathscr{M} \backslash \mathscr{A}\}=\sum_{\left\{N_{i}\right\}}\left(\Theta\left(E-\sum_{i=1}^{s} N_{i} \lambda_{i}\right) \delta_{\sum_{i=1}^{s} N_{i}, N} \Theta\left(S_{l}-\Delta\right) \Theta\left(S_{s-l}-\Delta\right)\right),
$$

где $\sum N_{i}=N$.

Здесь сумма берется по всем целым $N_{i}, \Theta(\lambda)$ - функция Хевисайда, а $\delta_{k_{1}, k_{2}}-$ символ Кронекера.

Используем интегральные представления:

$$
\begin{aligned}
& \delta_{N N^{\prime}}=\frac{e^{-\nu N}}{2 \pi} \int_{-\pi}^{\pi} d \varphi e^{-i N \varphi} e^{\nu N^{\prime}} e^{i N^{\prime} \varphi}, \\
& \Theta(y)=\frac{1}{2 \pi i} \int_{-\infty}^{\infty} d \lambda \frac{1}{\lambda-i} e^{\beta y(1+i \lambda)} .
\end{aligned}
$$


Прибегнем к стандартной регуляризации. Заменим первую $\Theta$ - функцию Хевисайда в (19), на непрерывную функцию

$$
\begin{aligned}
& \Theta_{\alpha}(y)= \begin{cases}0 & \text { при } \alpha>1, y<0, \\
1-e^{\beta y(1-\alpha)} & \text { при } \alpha>1, y \geqslant 0,\end{cases} \\
& \Theta_{\alpha}(y)= \begin{cases}e^{\beta y(1-\alpha)} & \text { при } \alpha<0, y<0, \\
1 & \text { при } \alpha<0, y \geqslant 0,\end{cases}
\end{aligned}
$$

где $\alpha \in(-\infty, 0) \cup(1, \infty)$ - параметр. Получим

$$
\Theta_{\alpha}(y)=\frac{1}{2 \pi i} \int_{-\infty}^{\infty} e^{\beta y(1+i x)}\left(\frac{1}{x-i}-\frac{1}{x-\alpha i}\right) d x
$$

Если $\alpha>1$, тогда $\Theta(y) \leqslant \Theta_{\alpha}(y)$.

Пусть $\nu<0$. Заменив $(20)$ и $(21)$ в (19), поменяв местами интегрирование и суммирование и переходя к пределу при $\alpha \rightarrow \infty$, мы получим оценку

$$
\begin{aligned}
& \mathscr{N}\{\mathscr{M} \backslash \mathscr{A}\} \leqslant\left|\frac{e^{-\nu N+\beta E}}{i(2 \pi)^{2}}\right| \\
& \times \int_{-\pi}^{\pi}\left[\exp (-i N \varphi) \sum_{\left\{N_{j}\right\}}\left(\exp \left\{\left(-\beta \sum_{j=1}^{s} N_{j} \lambda_{j}\right)+(i \varphi+\nu) N_{j}\right\}\right)\right] d \varphi \\
& \times \Theta\left(S_{l}-\Delta\right) \Theta\left(S_{s-l}-\Delta\right), \\
& \sum N_{i}=N,
\end{aligned}
$$

где $\beta$ и $\nu$ - такие действительные параметры, для которых ряд сходится.

Имеем

$$
\int_{0}^{\infty} d M \Theta\left(M-\sum_{i=1}^{s} N_{i} \lambda_{i}\right) e^{-\beta E}=\int_{\sum_{i=1}^{s} N_{i} \lambda_{i}}^{\infty} d E e^{-\beta E}=\frac{e^{-\beta \sum_{i=1}^{s} N_{i} \lambda_{i}}}{\beta} .
$$

Оценивая правую часть, пронеся модуль под интеграл и затем под знак суммы и интегрируя по $\varphi$, получим

$$
\mathscr{N}\{\mathscr{M} \backslash \mathscr{A}\} \leqslant \frac{e^{-\nu N+\beta E}}{2 \pi} \sum_{\left\{N_{i}\right\}} \exp \left\{-\beta \sum_{i=1}^{s} N_{i} \lambda_{i}+\nu N_{i}\right\} \Theta\left(S_{l}-\Delta\right) \Theta\left(S_{s-l}-\Delta\right) .
$$

Обозначим

$$
Z(\beta, N)=\sum_{\left\{N_{i}\right\}} e^{-\beta \sum_{i=1}^{s} N_{i} \lambda_{i}},
$$

где сумма берется по всем $N_{i}$ таким, что $\sum_{i=1}^{s} N_{i}=N$,

$$
\begin{gathered}
\zeta_{l}(\nu, \beta)=\prod_{i=1}^{l} \xi_{i}(\nu, \beta), \quad \zeta_{s-l}(\nu, \beta)=\prod_{i=l+1}^{s} \xi_{i}(\nu, \beta), \\
\xi_{i}(\nu, \beta)=\frac{1}{\left(1-e^{\nu-\beta \lambda_{i}}\right)^{q_{i}}}, \quad i=1, \ldots, l .
\end{gathered}
$$


Из неравенства для гиперболического косинуса $\operatorname{ch}(x)=\left(e^{x}+e^{-x}\right) / 2$ при $\left|x_{1}\right| \geqslant \delta$, $\left|x_{2}\right| \geqslant \delta$,

$$
\operatorname{ch}\left(x_{1}\right) \operatorname{ch}\left(x_{2}\right)=\operatorname{ch}\left(x_{1}+x_{2}\right)+\operatorname{ch}\left(x_{1}-x_{2}\right)>\operatorname{ch}\left(x_{1}+x_{2}\right)>\frac{e^{\delta}}{2}
$$

следует, что для всех положительных $с$ и $\Delta$ выполняется неравенство (ср. [10])

$$
\Theta\left(S_{s-l}-\Delta\right) \Theta\left(S_{l}-\Delta\right) \leqslant e^{-c \Delta} \operatorname{ch}\left(c \sum_{i=1}^{l} N_{i}-c \phi_{l}\right) \operatorname{ch}\left(c \sum_{i=l+1}^{s} N_{i}-c \bar{\phi}_{s-l}\right)
$$

где

$$
\phi_{l}=\sum_{i=1}^{l} \frac{q_{i}}{e^{\beta^{\prime} \lambda_{i}-\nu^{\prime}}-1}, \quad \bar{\phi}_{s-l}=\sum_{i=l+1}^{s} \frac{q_{i}}{e^{\beta \lambda_{i}-\nu}-1} .
$$

Получаем

$$
\begin{aligned}
& \mathscr{N}\{\mathscr{M} \backslash \mathscr{A}\} \leqslant e^{-c \Delta} \exp (\beta E-\nu N) \times \sum_{\left\{N_{i}\right\}} \exp \left\{-\beta \sum_{i=1}^{l} N_{i} \lambda_{i}+\nu \sum_{i=1}^{l} N_{i}\right\} \operatorname{ch}\left(\sum_{i=1}^{l} c N_{i}-c \phi\right) \\
& \quad \times \exp \left\{-\beta \sum_{i=l+1}^{s} N_{i} \lambda_{i}+\nu \sum_{i=l+1}^{s} N_{i}\right\} \operatorname{ch}\left(\sum_{i=l+1}^{s} c N_{i}-c \bar{\phi}\right) \\
&=e^{\beta E} e^{-c \Delta}\left(\zeta_{l}(\nu-c, \beta) \exp \left(-c \phi_{l}\right)+\zeta_{l}(\nu+c, \beta) \exp \left(c \phi_{l}\right)\right) \\
& \times\left(\zeta_{s-l}(\nu-c, \beta) \exp \left(-c \bar{\phi}_{s-l}\right)+\zeta_{s-l}(\nu+c, \beta) \exp \left(c \bar{\phi}_{s-l}\right)\right) .
\end{aligned}
$$

Используем равенства

$$
\left.\frac{\partial}{\partial \nu} \ln \zeta_{l}\right|_{\substack{\beta=\beta^{\prime} \\ \nu=\nu^{\prime}}} \equiv \phi_{l},\left.\quad \frac{\partial}{\partial \nu} \ln \zeta_{s-l}\right|_{\substack{\beta=\beta^{\prime} \\ \nu=\nu^{\prime}}} \equiv \bar{\phi}_{s-l}
$$

и разложение $\zeta_{l}(\nu \pm c, \beta)$ по формуле Тейлора. Существует $\gamma<1$ такое, что

$$
\ln \left(\zeta_{l}(\nu \pm c, \beta)\right)=\ln \zeta_{l}(\nu, \beta) \pm c\left(\ln \zeta_{l}\right)_{\nu}^{\prime}(\nu, \beta)+\frac{c^{2}}{2}\left(\ln \zeta_{l}\right)_{\nu}^{\prime \prime}(\nu \pm \gamma c, \beta)
$$

Подставляя это разложение и используя формулу (32), мы получим, что $\phi_{\nu, \beta}$ сокращается.

Другое представление формулы Тейлора дает

$$
\begin{aligned}
\ln \left(\zeta_{l}(\nu+c, \beta)\right)= & \ln \left(\zeta_{l}(\beta, \nu)\right)+\frac{c}{\beta} \frac{\partial}{\partial \nu} \ln \left(\zeta_{l}(\beta, \nu)\right) \\
& +\int_{\nu}^{\nu+c / \beta} d \nu^{\prime}\left(\nu+c / \beta-\nu^{\prime}\right) \frac{\partial^{2}}{\partial \nu^{\prime 2}} \ln \left(\zeta_{l}\left(\beta, \nu^{\prime}\right)\right) .
\end{aligned}
$$

Аналогично для $\zeta_{s-l}$. 
Из явного вида функции $\zeta_{l}(\beta, \nu)$ получаем

$$
\frac{\partial^{2}}{\partial \nu^{2}} \ln \left(\zeta_{l}(\beta, \nu)\right)=\beta^{2} \sum_{i=1}^{l} \frac{g_{i} \exp \left(-\beta\left(\lambda_{i}+\nu\right)\right)}{\left(\exp \left(-\beta\left(\lambda_{i}+\nu\right)\right)-1\right)^{2}} \leqslant \beta^{2} Q d,
$$

где $d$ выражается формулой

$$
d=\frac{\exp \left(-\beta\left(\lambda_{1}+\nu\right)\right)}{\left(\exp \left(-\beta\left(\lambda_{1}+\nu\right)\right)-1\right)^{2}}
$$

Та же оценка имеет место для $\zeta_{s-l}$.

Учитывая, что $\zeta_{l} \zeta_{s-l}=\zeta_{s}$, мы получим оценку при $\beta=\beta^{\prime}, \nu=\nu^{\prime}$

$$
\mathscr{N}\{\mathscr{M} \backslash \mathscr{A}\} \leqslant \zeta_{s}\left(\beta^{\prime}, \nu^{\prime}\right) \exp \left(-c \Delta+\frac{c^{2}}{2} \beta^{2} Q d\right) \exp \left(E \beta^{\prime}-\nu^{\prime} N\right) .
$$

Теперь выразим $\zeta_{s}\left(\nu^{\prime}, \beta^{\prime}\right)$ через $Z(\beta, N)$. Для этого докажем лемму.

ЛЕмма 2. При высказанных предположениях асимптотика интеграла

$$
Z(\beta, N)=\frac{e^{-\nu N}}{2 \pi} \int_{-\pi}^{\pi} d \alpha e^{-i N \alpha} \zeta_{s}(\beta, \nu+i \alpha)
$$

имеет вид

$$
Z(\beta, N)=C e^{-\nu N} \frac{\zeta_{s}(\beta, \nu)}{\left|\left(\partial^{2} \ln \zeta_{s}(\beta, \nu)\right) /\left(\partial^{2} \nu\right)\right|}\left(1+O\left(\frac{1}{N}\right)\right),
$$

где $C$ - const.

Имеем

$$
Z(\beta, N)=\frac{e^{-\nu N}}{2 \pi} \int_{-\pi}^{\pi} e^{-i N \alpha} \zeta_{s}(\beta, \nu+i \alpha) d \alpha=\frac{e^{-\nu N}}{2 \pi} \int_{-\pi}^{\pi} e^{N S(\alpha, N)} d \alpha,
$$

где

$$
S(\alpha, N)=-i \alpha+\ln \zeta_{s}(\beta, \nu+i \alpha)=-i \alpha-\sum_{i=1}^{s} q_{i} \ln \left[1-e^{\nu+i \alpha-\beta \lambda_{i}}\right] .
$$

Здесь $S$ зависит от $N$, поскольку от $N$ зависят $s, \lambda_{i}$ и $\nu$; последнее выбирается таким образом, чтобы точка $\alpha=0$ была стационарной точкой фазы $S$, т.е. из условия

$$
N=\sum_{i=1}^{s} \frac{q_{i}}{e^{\beta \lambda_{i}-\nu}-1} .
$$

Будем считать, что $a_{1} N \leqslant s \leqslant a_{2} N, a_{1}, a_{2}=$ const и, кроме того, $0 \leqslant \lambda_{i} \leqslant B, B=$ const, $i=1, \ldots, s$. При выполнении этих условий в некотором интервале $\beta \in\left[0, \beta_{0}\right]$ значений обратной температуры все производные фазы ограничены, стационарная точка невырождена и вещественная часть фазы вне окрестности нуля строго меньше ее значения в нуле минус некоторое положительное число. Поэтому при вычислении асимптотики интеграла можно заменить отрезок интегрирования $[-\pi, \pi]$ отрезком $[-\varepsilon, \varepsilon]$. Сделаем в этом интеграле замену переменной

$$
z=\sqrt{S(0, N)-S(\alpha, N)} .
$$


Она голоморфна в круге $|\alpha| \leqslant \varepsilon$ в комплексной $\alpha$-плоскости и имеет голоморфную обратную, если $\varepsilon$ достаточно мало. В результате получаем

$$
\int_{-\varepsilon}^{\varepsilon} e^{N S(\alpha, N)} d \alpha=e^{N S(0, N)} \int_{\gamma} e^{-N z^{2}} f(z) d z,
$$

где контур $\gamma$ в комплексной $z$-плоскости получается заменой $(42)$ из отрезка $[-\varepsilon, \varepsilon]$, а

$$
f(z)=\left.\left(\frac{\partial \sqrt{S(0, N)-S(\alpha, N)}}{\partial \alpha}\right)^{-1}\right|_{\alpha=\alpha(z)} .
$$

При малом $\varepsilon$ контур $\gamma$ целиком лежит внутри двойного сектора $\operatorname{re}\left(z^{2}\right)>c(\operatorname{re} z)^{2}$ для некоторого $c>0$, поэтому его можно "сдвинуть" на вещественную ось, не меняя интеграл с точностью до экспоненциально малых по $N$ членов. Итак, с указанной точностью имеем

$$
Z(\beta, N)=\frac{e^{-\nu N}}{2 \pi} \int_{-\varepsilon}^{\varepsilon} e^{-N z^{2}} f(z) d z .
$$

Теперь, поскольку переменная $z$ стала вещественной, функцию $f(z)$ можно считать финитной (изменив ее вне отрезка интегрирования), распространить интеграл на всю ось (что снова даст экспоненциально малую ошибку) и затем вычислить асимптотическое разложение интеграла, разлагая подынтегральную функцию в ряд Тейлора по $z$ с остаточным членом. Это обосновывает применение метода стационарной фазы к указанному интегралу для данного случая.

Лемма 3. Имеем

$$
\frac{1}{\mathscr{N}(\mathscr{M})} \sum_{\left\{N_{i}\right\}} e^{-\beta \sum_{i=1}^{s} N_{i} \lambda_{i}}
$$

где $\sum N_{i}=N, \lambda_{i} N_{i} \leqslant E-N^{1 / 2+\varepsilon}$, стремится $\kappa$ нулю быстрее $N^{-k}$ для любого $k$, $\varepsilon>0$.

Рассмотрим точку минимума по $\beta$ при $\nu(\beta, N)$, удовлетворяющем условию

$$
\sum \frac{q_{i}}{e^{\beta \lambda_{i}-\nu(\beta, N)}-1}=N
$$

Нетрудно убедиться, что она удовлетворяет условию (17). Предположим, что условие леммы не выполняется.

Тогда при $\sum N_{i}=N, \sum \lambda_{i} N_{i} \geqslant E-N^{1 / 2+\varepsilon}$

$$
e^{\beta E} \sum_{\left\{N_{i}\right\}} e^{-\beta \sum_{i=1}^{s} N_{i} \lambda_{i}} \geqslant e^{\left(N^{1 / 2}+\varepsilon\right) \beta},
$$

и очевидно, что $\beta \ll \frac{1}{\sqrt{N}}$ дает минимум (31) при выполнении леммы 1.

По той же схеме Коваль получил и прямое доказательство этой леммы.

Положим в формуле (36) после подстановки (38) $c=\Delta / N^{1+\alpha}$, тогда нетрудно видеть, что при $\Delta=N^{3 / 4+\varepsilon}$ отношение

$$
\frac{\mathscr{N}(\mathscr{M} \backslash \mathscr{A})}{\mathscr{N}(\mathscr{M})} \approx \frac{1}{N^{m}},
$$

где $m$ - любое целое. Теорема 1 доказана. 
ЗАмечание 1. В работе [13] по небрежности вкралась ошибка. На странице 7 в ссылке на работу [10] вместо формулы (95) следовало указать формулу (94) и рассмотреть формулу (96), а именно $\nu=\nu^{\prime}+O\left(1 / N^{1 / 4}\right)$. Это меняет оценку (4): экспонента должна быть $3 / 4$, а не $1 / 2$.

Случай 2) доказывается совершенно аналогично (ср. [10], [14]) за исключением того, что $\beta$ будет меньше нуля и $d$ из формулы (35) нужно заменить на

$$
d^{\prime}=\frac{\exp \left(-\beta\left(\lambda_{s}+\nu\right)\right)}{\left(\exp \left(-\beta\left(\lambda_{s}+\nu\right)\right)-1\right)^{2}} .
$$

Мы доказываем кумулятивную формулу, в которой плотности по виду совпадают с распределением Бозе-Эйнштейна. Отличие состоит только в том, что в формуле вместо набора $\lambda_{n}$ случайных величин или собственных значений оператора Гамильтониана стоят некоторые средние их значения по ячейкам. В силу нашей теоремы $\varepsilon_{i}-$ средние значения энергии $\lambda_{k}$ в $i$-ой ячейке - это нелинейные средние по Колмогорову.

ЗАмечаниЕ 2. Если нормировать $N_{i}$ в формуле (18), поделив на $N$, то правая часть (18) даст оценку $N^{-1 / 4+\varepsilon}$. Условие (19) также будет необходимо нормировать, поделив на $N$.

Тогда мы полагаем, что $Q=\sum_{i=1}^{s} q_{i} \rightarrow \infty$, и оценка будет $O\left(Q^{-1 / 4+\varepsilon}\right)$. Условия на $\beta$ и $\nu$ будут иметь вид

$$
\sum \frac{\lambda_{i} q_{i}}{e^{\beta \lambda_{i}-\nu}-1}=E^{0}, \quad \sum \frac{q_{i}}{e^{\beta \lambda_{i}-\nu}-1}=Q, \quad E^{0}=\frac{E}{Q} .
$$

ПримеР. Пусть задан временной ряд

$$
b_{1}, b_{2}, \ldots, b_{M}, \quad \sum_{i=1}^{M} b_{i}^{2}=A_{0} \gg 1 .
$$

Используя теорему Котельникова, получаем функцию вида

$$
f(t)=\sum_{n=1}^{M} a_{n} \cos \left(\frac{\pi n t}{M}\right)
$$

обращающуюся в целых точках $t=i$ в заданные значения $b_{i}, i=1, \ldots, M$. Будем предполагать, что

1) $(1 / M) \sum_{n=1}^{M} a_{n}^{2}=(1 / M) \sum_{i=1}^{M} b_{i}^{2}=A_{0}, \quad A_{0} M \gg 1$;

2) $a_{n}$ - рациональные числа;

3) энергия ограничена, а именно

$$
\int_{0}^{2 \pi}\left(\frac{d f}{d t}\right)^{2}(t) d t \leqslant E, \quad E \gg 1 .
$$

Отсюда следует

$$
\sum_{n=1}^{M} n^{2} a_{n}^{2} \leqslant \frac{E M^{2}}{\pi^{2}}
$$


Cпектралъной плотностъю интервала $l$ называется [15] средний квадрат амплитуды, а именно

$$
A_{l}=\frac{1}{M-l} \sum_{n=l}^{M} a_{n}^{2} .
$$

Поскольку в силу 1) $a_{n}^{2}$ - рациональные числа, то их можно привести к общему знаменателю $N$ так, что $a_{n}^{2}=N_{n} / N$, где $N_{n}$ и $N$ - целые. Тогда получим

$$
\sum_{n=1}^{M} \frac{N_{n}}{N}=A_{0} M
$$

Далее положим $\lambda_{n}=n^{2}$, тогда условие (48) примет вид

$$
\sum_{n=1}^{M} \lambda_{n} \frac{N_{n}}{N} \leqslant \frac{E M^{2}}{\pi^{2}} .
$$

Теперь условия 1), 2), 3) принимают обычной вид для теоремы 1. Мы будем предполагать, что наборы $\left\{N_{n} / N\right\}_{n=1}^{M}$ равновероятны при ограничениях (49) u (50).

Отсюда, поскольку

$$
A_{l}=\frac{1}{M-l} \sum_{n=l}^{M} \frac{N_{n}}{N},
$$

то, если $D$ известно, то в силу теоремы 1

$$
A_{l} \cong \frac{1}{M-l} \sum_{n=l}^{M} \frac{\rho_{n}(D)}{e^{\pi^{2} n^{2} \beta-\sigma}-1}
$$

при условиях

$$
\begin{aligned}
& \sum_{n=1}^{M} \frac{n^{2} \rho_{n}(D)}{e^{\pi^{2} n^{2} \beta-\sigma}-1}=\frac{E M^{2}}{\pi^{2}}, \\
& \sum_{n=1}^{M} \frac{\rho_{n}(D)}{e^{\pi^{2} n^{2} \beta-\sigma}-1}=A_{0} M .
\end{aligned}
$$

Для колмогоровской турбулентности $D=-5 / 3$, а условие $A_{0} \gg 1$ означает сильную внешнюю подкачку. Турбулентность квантовой жидкости характеризуется тем, что вихри могут образовываться лишь дискретным образом, подчиняясь условиям квантования, зависящим от постоянной Планка $h$. Возможно, что в этом случае условия Колмогорова на спектральную плотность должны быть проквантованы согласно формулам (52)-(54).

В заключение выражаю глубокую благодарность В. В. Вьюгину, А.Н. Соболевскому и Е. В. Щепину за ценные дискуссии. 


\section{СПИСОК ЦИТИРОВАННОЙ ЛИТЕРАТУРЫ}

[1] Математический энииклопедический словаръ, ред. Ю.В.Прохоров, Советская энциклопедия, М., 1988.

[2] Yu. I. Manin, The Notion of Dimension in Geometry and Algebra, arXiv:math.AG/ 0502016.

[3] В.П. Маслов, "Отрицательная асимптотическая топологическая размерность, новый конденсат и их связь с квантованным законом Ципфа", Матем. заметки, 80:6 (2006), $856-863$.

[4] В. П. Маслов, "Общее понятие топологических пространств отрицательной размерности и квантованние их плотностей.", Матем. заметки, 81:1 (2007), 157-160.

[5] V.P. Maslov, Negative Dimension in General and Asymptotic Topology, arXiv: math.GM/ 0612543.

[6] V.P. Maslov, Dimension of Holes and High-Temperature Condensate in Bose-Einstein Statistics, arXiv: physics/0612182.

[7] V. P. Maslov, "Quantum Linguistic Statistics", Russ. J. Math. Phys., 13:3 (2006), 315-325.

[8] V.P. Belavkin, V.P. Maslov, "Design of the optimal dynamic analyzer: mathematical aspects of sound and visual pattern recognition", Mathematical Aspects of Computer Engineering, eds. V. P. Maslov, K. A. Volosov, Mir, Moscow, 146-237.

[9] G. Shafer, V. Vovk, Probability and Finance. It's Only a Game!, Wiley Series in Probability and Statistics, Wiley, New York, 2001.

[10] В.П. Маслов, "Нелинейное среднее в экономике", Матем. заметки, 78:3 (2005), 377-395.

[11] В. П. Маслов, "Об одной общей теореме теории множеств, приводящей к распределению Гиббса, Бозе-Эйнштейна, Парето и закону Ципфа-Мандельброта для фондового рынка", Матем. заметки, 78:6 (2005), 870-877.

[12] В. П. Маслов, "Закон "отсутствия предпочтения" и соответствующее распределение в частотной теории вероятностей", Матем. заметки, 80:2 (2006), 220-230.

[13] V.P. Maslov, On a General Theorem of Number Theory Leading to the Gibbs, Bose-Einstein, and Pareto Distribution as well as to the Zipf-Mandelbrot Law for the Stock Market, arXiv: physics/0601005.

[14] V. P. Maslov, T. V. Maslova, Rank Distributions in Semiotics, arXiv: math.PR/0612540.

[15] У. Фриш, Турбулентность. Наследие А.Н. Колмогорова, ФАЗИС, М., 1998.

В. П. Маслов

Московский государственный университет

им. М. В. Ломоносова

E-mail: v.p.maslov@mail.ru
Поступило

07.01 .2007 